\title{
Hekzakopter insansız hava aracı için eşdeğer bozucu etki tahmini kullanılarak bozucu etki önleyici denetleyici tasarımı
}

\section{Anti-disturbance controller design for a hexacopter unmanned aerial vehicle using equivalent disturbance estimation}

\author{
Hasan Başak 1,* (D) Emre Kemer ${ }^{2}$ iD \\ ${ }^{1}$ Artvin Çoruh Üniversitesi, Mühendislik Fakültesi, Elektrik-Elektronik Mühendisliği Bölümü, Artvin, Türkiye \\ ${ }^{2}$ Uşak Üniversitesi, Mühendislik Fakültesi, Elektrik-Elektronik Mühendisliği Bölümü, Uşak, Türkiye
}

\begin{abstract}
Özet
İnsansız Hava Araçları (IHHA'lar) video kamera, fotoğraf makinesi, lazer tarama cihazı, vb. araçlar ile donatılmıș ve görevlerini uzaktan kumandalı veya otonom olarak icra edebilmektedirler. Hızla gelişmekte olan bilgisayarın veri işleme kapasitesi, sensör ve yarı iletken teknolojileri küçük boyutlu döner kanatlı İHA'ların tasarlanmasina imkân sağlamaktadır. Küçük boyutlu döner kanatlı İHA sistemleri, mevcut otonom denetim yöntemlerinin henüz yeterli seviyeye gelememesinden dolayi, ani rüzgâr durumu gibi rastgele çevresel etkenler ve dişarıdan gelen değişik karakteristik özelliklere sahip bozucu etkilere karşı yeterli düzeyde dayanıklı değildirler. Bu çalışmada hekzakopter İHA sistemlerinin bozucu etkililere rağmen kararlılığını koruyup görevini etkin bir şekilde yerine getirmesi amacıyla bozucu etki önleyici denetleyici tasarlanmıştır. İHA sisteminin yönelim kararlılığı için lineer kuadratik regülatör yöntemi kullanılarak dayanıklı geri besleme kazancı hesaplanmıştır. Ayrıca gözlemci kazancı ve alçak geçiren filtreden oluşan bozucu etki eşdeğerini tahmin mekanizması da tasarlanmıştır. Bu yapı sistemdeki bilinmeyen bozucu etkilerin zamanında tahmin edilmesine olanak sağlamaktadır. Tasarlanan bozucu etki önleyici denetleyicisi kapalı çevrim İHA sisteminin gürbüz kararlılığını garanti eder. Benzetim sonuçları hekzakopter İHA için tasarlanan bozucu önleyici denetleyicinin etkinliğini göstermiştir.
\end{abstract}

Anahtar kelimeler: Hekzakopter İHA, Bozucu etki önleyici denetleyici, Lineer kuadratik regülatör, Eşdeğer bozucu etki tahmini

\section{Giriş}

İnsansiz hava arac1 (IHA) sistemleri askeri uygulamaların yanı sıra son yıllarda bilgisayar ve sensör teknolojisindeki gelişmeler nedeniyle, popülerliği giderek artan sivil uygulama alanları da bulmaktadır. İHA, özellikle askeri uygulamalar başta olmak üzere doğal afetlerde yardım ve kurtarma, çeşitli spor faaliyetlerinin izlenmesi, hasar tespiti ve değerlendirmesi, haritalama işlemleri, orman yangınlarının tespiti ve ilk müdahalesi, petrol ve doğal gaz boru hatlarının gözlenmesi ve güvenliği, trafik denetimi, kaçak yapılaşmanın kontrolü vb. uygulamaların hızlı ve

\begin{abstract}
Unmanned Aerial Vehicles (UAVs) equipped with video camera, camera, laser scanning device, etc. and perform their duties remotely or automatically. Recently, rapid progress in development of computers' data processing capacities, sensors, and semiconductor technologies have resulted in the emergence of small-sized rotary-wing UAVs. Small size rotary-wing UAV systems are more sensitive to environmental conditions such as gust and external disturbances because existing autonomous control systems are not sufficient to cope with these disturbances. In this study, an anti-disturbance controller has been designed for that a hexacopter UAV maintains its stability and are able to perform their duties in the despite of the external disturbances. Optimal gain has been calculated by linear quadratic regulator method for the attitude stability of the UAV system. In addition, a mechanism of equivalent disturbance estimation, which is consisting of an observer gain and a low pass filter, is designed. This structure estimated the unknown disturbing effects in the UAV system in time. The designed anti-disturbance controller guarantees the robust stability of the closed-loop UAV system. The simulation results demonstrated the effectiveness of the designed anti-disturbance controller for the hexacopter UAV.
\end{abstract}

Keywords: Hexacopter UAV, Anti-disturbance control, Linear quadratic regulator, Equivalent disturbance estimation

güvenli bir şekilde gerçekleştirilmesine olanak sağlamaktadır. Bu kullanım avantajlarının yanında insansız hava araçlarının, insanlı olanlara nazaran teknik anlamdaki en önemli dezavantaj1, mevcut otonom denetim sistemleri henüz yeterli seviyeye gelmediği için, ani rüzgâr durumu gibi meteorolojik şartlara karşı daha hassas olmaları ve taşıyabilecekleri faydalı yükteki belirsizliklerin daha sınırlı olmasıdır. Olası uygulama alanlarının genişlemesi sebebiyle zorlu çevresel şartlar ve karmaşık görevlerle başa çıkabilen insansız sistemleri tasarlamak için denetim ve navigasyon alanlarında yeni talepler meydana gelmiştir. Döner kanatlı

* Sorumlu yazar / Corresponding author, e-posta / e-mail:hasanbasak@ artvin.edu.tr (H. Başak)

Geliş / Recieved: 04.10.2020 Kabul/ Accepted: 05.03.2021 Yayımlanma / Published: 27.07.2021

doi: 10.28948/ngmuh.804951 
insanız hava araçları dikey kalkış-iniş, agresif hareket yapabilme özelliklerinden dolayı birçok uygulamanın en iyi çözümü olarak karşımıza çıkmaktadır. Fakat döner kanatlı insansız hava araçları otonom ve etkili uçuş yapabilmeleri için önemli mühendislik sorunlarını da içerisinde barındırmaktadırlar [1]. Düşük eylemsizlik momenti ve karıșık aerodinamik etkilere maruz kalınması araçların uçuşunu etkilemesi sebebiyle bu tip insansız hava araçlarının agresif dinamikleri ile karakterize edilmesi gerekmektedir. Sonuç olarak, bu sistemlerin otonom denetimi zor bir konudur. Ĕger atmosferik bozucu etkiler de dikkate alınırsa, bu sorun daha da zorlu hale gelir. Bu nedenle, yeni denetim sistemleri tasarlarken sistemin maruz kalacağı bozucu etkiler göz önünde bulundurmalı ve bu perspektifte etkili denetim yaklaşımları geliștirilmelidir.

Bozucu etkilerin azaltılması veya ortadan kaldırılması için klasik geri beslemeli denetleyici tasarımı önerilebilir. Ancak, klasik geri beslemeli denetleyici tasarımları birçok tasarım kısıtlamalarına sahiptir ve bu tek derece bağımsız denetleyici yapısı olarak bilinir. Gerçek şu ki ölçülemeyen bozucuların etkilerinin zayıflatılması için öncelikli olarak geri beslemeli denetleyici tasarlanması zorunludur. Fakat denetleyici tasarımında örneğin; kararlılık, performans, referans takip etme, düzenleme, bozucu etki reddi ve dayanıklılık gibi birçok tasarım gereksinimleri vardır. Diğerlerinin içinden en önemli olan gereksinimlerden; takip etme, bozucu etkinin reddi ve gürbüz performans gibi kısıtlamalarından herhangi birini sağlamak için diğerlerinden fedakârlık etmek gerekir. Burada bozucu etki önleyici yöntemler kısıtlamaları azaltmada etkin bir yöntem olacaktır [2].

Hekzakopter İHA'larının yönelim ve yörünge takibi üzerine farklı yöntemler literatürde sunulmuştur. Hekzakopter yörünge izleme problemini çözmek için, [3] çalışmasında doğrusal olmayan gürbüz ve adaptif geri adımlamalı denetim stratejisi hiyerarşik olarak önerilmektedir. Derawi ve diğerleri [4] çalışmasında gürültü ve belirsizliklerin yönelim üzerindeki etkisini azaltmak için gürbüz durum geri-beslemeli denetleyici geliştirmiştir. Oransal-İntegral-Türevsel (PID) denetleyicisi hekzakopterin yörünge takibi için [5] çalışmasında sunulmuştur. Ayrıca çalışma [6]'da PID denetleyicisi ile yuvarlanma, yunuslama, yönelme ve irtifanın dengelenmesi sağlanmış ve motorda oluşacak arızaların tespiti ve izolasyonu yapılmıştır. Yönelim takibi için, çalışma [7] adaptif yapay sinir ağları kullanılarak PID denetleyici tasarımını sunmuştur. Ligthart ve diğerleri [8] bir İHA sistemin kararlığını ve güvenliğini sağlamak için model öngörülü denetleyici tasarımı sunmuştur. Hekzakopter İHA için, [9] çalışmasında hataya toleranslı denetleyici tasarımı verilmiştir ve burada dayanıklı denetleyicileri hata durumuna göre programlanmıştır. Son zamanlarda İHA'lar için bozucu etkileri dışlayıcı denetleyici tasarımı dikkat çeken bir çalışma konusudur. Örneğin, Guo ve diğerleri [10] bozucu etki ve genişletilmiş durum gözlemcileri kullanarak dört motorlu İHA'da yük ve rüzgârdan dolayı oluşacak bozucu etkileri elimine etmek için denetim geliştirmişlerdir. Benzer şekilde rüzgârın etkilerini tahmin etmek için genişletilmiş durum gözlemciler ve geri adımlama yöntemi kullanılarak çalışma [11]'de aktif denetleyici tasarlanmıştır. Yuan ve diğerleri [12] çalışmasında doğrusal olmayan genişletilmiş durum gözlemci ve doğrusal olmayan kompozit denetleyici yöntemlerini İHA yönelim takibi için önermiştir. Sonuç olarak, farklı denetleyici tasarlama yöntemleri ile bozucu etki tahminleri birleştirilerek farklı performans ihtiyaçlarına cevap verilebilmektedir.

$\mathrm{Bu}$ çalışmada, lineer kuadratik regülatör yöntemi ve eşdeğer bozucu etki tahmini yöntemi [13] birleştirilerek, altı motorlu İHA sisteminin dışarıdan maruz kalabileceği bozucu etkileri elimine etmek amaçlanmıştır. Dışarıdan gelebilecek bozucular direk olarak ölçülememesi veya ölçmek için çok pahalı araçlar gerekli olması sebebiyle ölçülebilen değişkenler kullanılarak bozucu etkiler tahmin edilebilir. Bozucu etkiler eşdeğer bozucu etki tahmini yöntemi kullanılarak sistem girişindeki bozucu etkiler tahmin edilecektir. Böylece sistem girişindeki bozucu etiklerin dışlanması sağlanacaktır. Benzetim sonuçları önerilen denetim yapısının geçerliliğini göstermektedir.

\section{Materyal ve metot}

\subsection{Hekzakopter insansız hava aracı matematiksel modeli}

Altı rotordan oluşan döner kanatlı İHA esnemez bir gövdeye sahip olduğu varsayılır. Rotorlar Şekil l'de gösterildiği gibi gerekli kuvvetleri ve torkları üretir. Tüm rotorlar 1 ile 6 arası etiketlenmiştir, burada tek numaralı (1, 3 ve 5) rotorlar saat yönünün tersine ve çift sayı (2, 4 ve 6$)$ saat yönünde döner. Aracın yüksekliği, rotor hızlarını eşit miktarda artırıp-azaltarak değiştirilebilir. 4 ve 5 nolu rotorların hızının artırılması ve aynı zamanda 1 ve 2 nolu rotorların hızları azaltılması, ileri hareketin yapılmasını sağlar. Ayrıca $(1,5,6)$ ve $(2,3,4)$ nolu rotor gruplarının hızlarını artırıp-azaltarak yuvarlanma hareketi oluşur ve bu y-ekseni boyunca yanal hareketinin de oluşmasını sağlar. Son olarak, rotorlar $(1,3,5)$ saat yönünün tersine, rotorlar $(2$, 4, 6) ters yönde dönerek yönelme (sapma) hareketi ayarlanmasını sağlar. İHA'nın dinamiğini tanımlamak için $\{B\}$ gövde ve $\{I\}$ eylemsizlik çerçevelerini inceleyelim (bkz. Şekil 1). Gövde çerçevesi Şekil 1'de belirtilirken $X_{B}-Y_{B}$ $Z_{B}$ düzlemi yüzeyine yerleştirilir ve hekzakopter'in ağırlık merkezi gövdenin merkezine yerleştirilir. Yönelim vektörü $\eta=(\Phi \theta \psi)^{T}$ ile ifade edelir burada $\Phi, \theta$ ve $\psi$ sirasiyla yuvarlanma, yunuslama ve yönelme açılarını göstermektedir. Aracın, eylemsizlik çerçevesindeki konum vektörü $\xi=\left(\begin{array}{lll}x_{I} & y_{I} & z_{I}\end{array}\right)^{T}$ ile ifade edilir.

Kuvvetlerin gövde çerçevesinden eylemsizlik çerçevesine dönüşümü, aşağıdaki $R_{f}$ döndürme matrisi kullanılarak yapılır:

$$
\begin{array}{ccc}
C \theta C \psi & S \Phi S \theta C \psi-C \Phi S \psi & C \Phi S \theta C \psi+S \Phi S \psi \\
\mid C \theta S \psi & S \Phi S \theta S \psi+C \Phi C \psi & C \Phi S \theta s \psi-S \Phi C \psi \mid \\
-S \theta & S \Phi C \theta & C \Phi C \theta
\end{array}
$$

Burada C(.) ve S(.) sirayla cos(.) ve sin(.) fonksiyonlarını ifade etmektedir. Açısal hızların gövde çerçevesinden eylemsizlik çerçevesine dönüşümü için aşağıdaki matris kullanılır:

$$
W_{\eta}^{-1}=\left|\begin{array}{ccc}
1 & \sin \Phi \tan \theta & \cos \Phi \tan \theta \\
0 & \cos \Phi & -\sin \Phi \\
0 & \sec \theta \sin \Phi & \cos \Phi \sec \theta
\end{array}\right|
$$




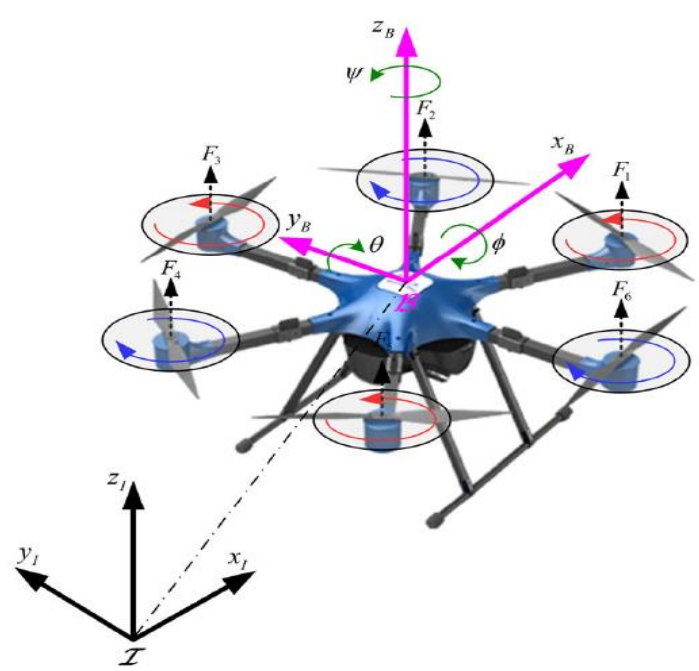

Şekil 1. Hekzakopter insansız hava aracının şematik diyagramı [3].

Alt1 rotorlu İHA'nın dinamik modeli, Newton-Euler formülazyonu kullanılarak aşağıdaki şekilde verilir [3],[5]:

$$
\begin{gathered}
\dot{\xi}=v \\
m \dot{v}=-m g e_{z}+R_{f} T_{f} \\
\dot{\eta}=W_{\eta}^{-1} \omega \\
J \dot{\omega}=-\omega \times J \omega+\tau_{f}
\end{gathered}
$$

Burada $v$ aracın eylemsizlik çerçevesindeki lineer hızıdır, $e_{z}=(0,0,1)^{T}$ z-ekseni boyunca bir vektörü temsil eder, $\omega$ açısal hızdır ve $J=\operatorname{diag}\left(I_{x x}, I_{y y}, I_{z z}\right)$ eylemsizlik matrisidir.

$T_{f}=\left(\begin{array}{lll}0 & 0 & u_{1}\end{array}\right)^{T}$ ve $\tau_{f}=\left(\begin{array}{lll}u_{2} & u_{3} & u_{4}\end{array}\right)^{T}\{B\}$ - çerçevesindeki kuvvet ve momentlerdir ve buradaki $u_{1-4}$ aşağıdaki gibi verilir:

$$
\begin{gathered}
u_{1}=F_{1}+F_{2}+F_{3}+F_{4}+F_{5}+F_{6} \\
u_{2}=-\frac{1}{2} F_{1} l+\frac{1}{2} F_{2} l+F_{3} l+\frac{1}{2} F_{4} l-\frac{1}{2} F_{5} l-F_{6} l \\
u_{3}=-\frac{\sqrt{3}}{2} F_{1} l-\frac{\sqrt{3}}{2} F_{2} l+\frac{\sqrt{3}}{2} F_{4} l+\frac{\sqrt{3}}{2} F_{5} l \\
u_{4}=\frac{d}{b}\left(-F_{1}+F_{2}-F_{3}+F_{4}-F_{5}+F_{6}\right)
\end{gathered}
$$

Burada $i$. rotor tarafindan üretilen kuvvet $F_{i}=\mathrm{b} \Omega_{i}^{2}$ olarak ifade edilir, $b$ pervanelerin itime katsayısı, $d$ sürüklenme katsayısı ve $\Omega_{i}, i$. rotor dönme hızını temsil etmektedir.

Denklem (3)-(6)'da ifadeler yerlerine konulursa, hekzakopter İHA'nın yönü ve öteleme hareketi şu şekilde türetilebilir:

$$
\begin{gathered}
\ddot{x}_{I}=(\cos \phi \sin \theta \cos \psi+\sin \phi \sin \psi) \frac{1}{m} u_{1} \\
\ddot{y}_{I}=(\cos \phi \sin \theta \sin \psi-\sin \phi \cos \psi) \frac{1}{m} u_{1} \\
\ddot{z}_{I}=-g+\cos \phi \cos \theta \frac{1}{m} u_{1} \\
\ddot{\phi}=\dot{\theta} \dot{\psi}\left(\frac{I_{y y}-I_{z z}}{I_{x x}}\right)+\frac{1}{I_{x x}} u_{2}
\end{gathered}
$$

$$
\begin{aligned}
& \ddot{\theta}=\dot{\phi} \dot{\psi}\left(\frac{I_{z z}-I_{x x}}{I_{y y}}\right)+\frac{1}{I_{y y}} u_{3} \\
& \ddot{\psi}=\dot{\theta} \dot{\phi}\left(\frac{I_{x x}-I_{y y}}{I_{z z}}\right)+\frac{1}{I_{z z}} u_{4}
\end{aligned}
$$

Denetim tasarımı kolaylaştırmak için Denklem (11)-(16) ile tanımlanan İHA modeli doğrusal olmayan durum-uzay gösterimi $\dot{x}=f(x(t), u(t))$ biçiminde ifade edilebilir ve buradaki $x$, durum-uzay ve $u$, kontrol giriş vektörleri aşağıdaki şekilde tanımlanır:

$$
\begin{aligned}
& x=\left[\begin{array}{lllll}
x_{1} & x_{2} & x_{3} & \ldots & x_{12}
\end{array}\right]^{T}
\end{aligned}
$$

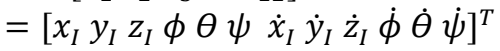

$$
\begin{aligned}
& u=\left[\begin{array}{llll}
u_{1} & u_{2} & u_{3} & u_{4}
\end{array}\right]^{T}
\end{aligned}
$$

Durum-uzay gösterimi Denklem (19)-(30), aşağıdaki gibi elde edilir:

$$
\begin{gathered}
\dot{x}_{1}=x_{7} \\
\dot{x}_{2}=x_{8} \\
\dot{x}_{3}=x_{9} \\
\dot{x}_{4}=x_{10} \\
\dot{x}_{5}=x_{11} \\
\dot{x}_{6}=x_{12} \\
\dot{x}_{7}=\left(\cos x_{4} \sin x_{5} \cos x_{6}+\sin x_{4} \sin x_{6}\right) \frac{1}{m} u_{1} \\
\dot{x}_{8}=\left(\cos x_{4} \sin x_{5} \sin x_{6}-\sin x_{4} \cos x_{6}\right) \frac{1}{m} u_{1} \\
\dot{x}_{9}=-g+\cos x_{4} \cos x_{5} \frac{1}{m} u_{1} \\
\dot{x}_{10}=x_{11} x_{12}\left(\frac{I_{y y}-I_{z z}}{I_{x x}}\right)+\frac{l}{I_{x x}} u_{2} \\
\dot{x}_{11}=x_{10} x_{12}\left(\frac{I_{z z}-I_{x x}}{I_{y y}}\right)+\frac{l}{I_{y y}} u_{3} \\
\dot{x}_{12}=x_{11} x_{10}\left(\frac{I_{x x}-I_{y y}}{I_{z z}}\right)+\frac{l}{I_{z z}} u_{4}
\end{gathered}
$$

Alt1 rotorlu İHA sisteminde bulunan tork ve kuvvet, sadece dört girişi $\left(u_{1}, u_{2}, u_{3}, u_{4}\right)$ oluşturur bundan dolayı bu girişler ile altı adet çıkışı $\left(x_{I}, y_{I}, z_{I}, \phi, \theta, \psi\right)$ kontrol etmek imkânsızdır. Pozisyon (Denklem (3)-(4)) ve yönelim (Denklem (5)-(6)) dinamikleri arasındaki etkileşim göz önüne alındığında, pozisyon hareketleri $\left(x_{I}-y_{I}\right)$ dolaylı olarak yönelim hareketleri $(\phi, \theta)$ ile kontrol edilebilir. Yani, toplam kaldırma kuvvetleri ile birlikte yönelimler, $\{x, y, z\}$ eksenlerinde bileşik kuvvetleri üretirler buda İHA'nın $x-y-z$ kartezyen koordinatlarındaki hareketleri sağlanmasına katkıda bulunur. Bundan dolayı bu çalışmada, İHA'nın belirtilen yörüngeyi takip etmesi için, bu harekete karşılık gelen yönelim yuvarlanma ve yunuslama hareketinin öncelikli olarak İHA model tarafindan yapması sağlanacaktır.

Doğrusal model için, durum-uzay denklemi durum $x_{e q}=$ 0 ve giriş $u_{e q}=\left[\begin{array}{llll}m g & 0 & 0 & 0\end{array}\right]$ denge noktasında Denklem (31)-(32) formunda verilir. 


$$
\begin{gathered}
\dot{x}=A x+B u \\
y=C x=\left[\begin{array}{llll}
\phi \theta & \psi & z
\end{array}\right]^{T}
\end{gathered}
$$

Buradaki $A$ ve $B$ matrisler aşağıdaki şekilde hesaplanır:

$$
\begin{gathered}
A=\frac{\partial f}{\partial x}=\left[\begin{array}{ccc}
\left.\frac{\partial f_{1}}{\partial x_{1}}\right|_{x_{e}, u_{e}} & \cdots & \left.\frac{\partial f_{1}}{\partial x_{n}}\right|_{x_{e}, u_{e}} \\
\vdots & \ddots & \vdots \\
\left.\frac{\partial f_{n}}{\partial x_{1}}\right|_{x_{e}, u_{e}} & \cdots & \left.\frac{\partial f_{n}}{\partial x_{n}}\right|_{x_{e}, u_{e}}
\end{array}\right], \\
B=\frac{\partial f}{\partial u}=\left[\begin{array}{ccc}
\left.\frac{\partial f_{1}}{\partial u_{1}}\right|_{x_{e}, u_{e}} & \cdots & \left.\frac{\partial f_{1}}{\partial u_{m}}\right|_{x_{e}, u_{e}} \\
\vdots & \ddots & \vdots \\
\left.\frac{\partial f_{n}}{\partial u_{1}}\right|_{x_{e}, u_{e}} & \cdots & \left.\frac{\partial f_{n}}{\partial u_{m}}\right|_{x_{e}, u_{e}}
\end{array}\right]
\end{gathered}
$$

\subsection{Bozucu etki önleyici denetleyici tasarımı}

\subsubsection{Lineer kuadratik regülatör}

Denklem (31)-(32)'de verilen İHA sistemi için geribeslemeli denetleyici Lineer Kuadratik Regülatör (LQR) yöntemi ile tasarlanmıştır. Bu yöntem sistem dinamiklerinin kuadratik maliyet fonksiyonunu en aza indirgemeye odaklanır. Kuadratik maliyet fonksiyonu:

$$
J=\int_{0}^{\infty}\left(x^{T} Q x+u^{T} R u\right) d t
$$

ile tanımlanır. Burada $Q$ ve $R$ durum ve giriş vektörleri için ağırlık matrisleridir. $Q$ ve $R$ matrisleri tasarımcı tarafindan seçilir ve bu şekilde maliyet fonksiyonu $J$ en aza indirgemeye çalışır. $J$ mümkün olduğu kadar azaltılırsa, $\lim _{t \rightarrow \infty} x=0$ sağlanır. Bu kapalı-döngü sistemin kararlı olmasını garanti eder.

Optimal denetim kuralı Denklem (35)'de verilmiştir.

ve

$$
u=-K x
$$

$$
K=R^{-1} B^{T} P
$$

burada $P$ ise aşağıda verilen Riccati denklemi çözümünden elde edilir:

$$
Q+P A+A^{T} P-P B R^{-1} B^{T} P=0
$$

Herhangi bir pozitif tanımlı $Q$ matrisi için eğer pozitif tanımlı $P$ matrisi var ve bu matris Lyapunov matris denklemini,

$$
A^{T} P+P A+Q=0 \text { veya } A^{T} P+P A<0,
$$

sağlıyorsa kapalı döngü sistem $\dot{x}=A x$ kuadratik kararlıdır. Ayrıca sistem için $V=x^{T} P x$ geçerli bir kuadraitk Lyapunov fonksiyonudur çünkü $P>0 \rightarrow V>0$ ve $\dot{V}=$ $x^{T}\left(A^{T} P+P A\right) x<0$.

$$
\begin{aligned}
& \text { Lyapunov fonksiyonun türevi, } \\
& \dot{V}=x^{T} A^{T} P x+x^{T} P A x+u^{T} B^{T} P x+x^{T} P B u \\
& =x^{T} P\left(A-B R^{-1} B^{T} P\right) x+x^{T}\left(A-B R^{-1} B^{T} P\right)^{T} P x
\end{aligned}
$$

$$
\text { (1) }
$$

ve $\mathrm{u}=-R^{-1} B^{T} P x$ olarak düşünülürse Riccati denklemi Denklem (39)'daki gibi yazılabilir:

$$
\begin{gathered}
P\left(A-B R^{-1} B^{T} P\right)+\left(A-B R^{-1} B^{T} P\right)^{T} P \\
=-Q-P B R^{-1} B^{T} P \\
\dot{V}<0
\end{gathered}
$$

Denklem (40), sistemin Lyapunov kararlılığını garanti eder [14].

\subsubsection{Q ve $R$ ă̆ırlık matrislerinin seçimi}

Pozitif tanımlı $Q$ ve $R$ matrisleri, kapalı-döngü sisteminin kutuplarının s-düzlemindeki dağılımı incelenerek seçilir. $Q$ matrisi durum değişkenlerinin üzerine kısıtlamaları belirler. Diğer taraftan $R$ matrisi sistem girişlerini belirler. Örneğin büyük değerler $Q$ matrisinde kapalı-döngü sisteminin kutuplarının daha sola yerleştirecek buda kapalı-döngü durumlarının daha hızlı cevap vermesini sağlayacaktır. Daha büyük $R$ değeri kontrol girişin daha çok kısıtlanmasına ve kutupların sanal eksene yakınlaşmasına neden olur. $\mathrm{Bu}$ matrisler köşegen seçilebilir fakat $R$ matrisin her bir elementi pozitif ve $Q$ matrisinin her bir elementi pozitif veya bazı elementleri sıfır seçilebilir [15].

\subsubsection{Sistem girişindeki bozucu etkilerin eşdeğer yaklaşımı ile tahmini}

$\mathrm{Bu}$ bölümde bozucu etkileri dişlamak için sistem girişindeki bozucu etkilerin eşdeğer yaklaşımı kullanılacaktır. $\mathrm{Bu}$ yöntemin bazı üstünlükleri şunlardır [13,16]: (a) bozucu etkileri önceden bilmek gerekmez, (b) ölçülen değişkenlerin türevi gerekmez, (c) sistem dinamiklerinin tersine gerek yoktur ve bundan dolayı kararlı olmayan kutuplar/ sıfırlar silinmesi önlenmiş olunur.

Aşağıdaki verilen lineer zamanla değişmeyen sistemi düşünürsek:

$$
\begin{gathered}
\dot{x}(t)=A x(t)+B(u(t)+d(t)) \\
y(t)=C x(t)
\end{gathered}
$$

burada $A \in \mathbb{R}^{n \times n}, B \in \mathbb{R}^{n \times n_{u}}$ ve $C \in \mathbb{R}^{n_{y} \times n}$ sistem matrisleridir. Bozucu etkiler sadece kontrol girişinden uygulandığını farz edilmiştir. Durum gözlemci Denklem (42)'de verilmiştir.

$$
\dot{\hat{x}}(t)=A \hat{x}(t)+B u_{f}(t)+L(y(t)-C \hat{x}(t))
$$

Burada $L \in \mathbb{R}^{n \times 1}$ gözlemci kazancıdır. Giriş bozucu etki eşdeğer tahmini Denklem (43)'deki gibi verilir. 


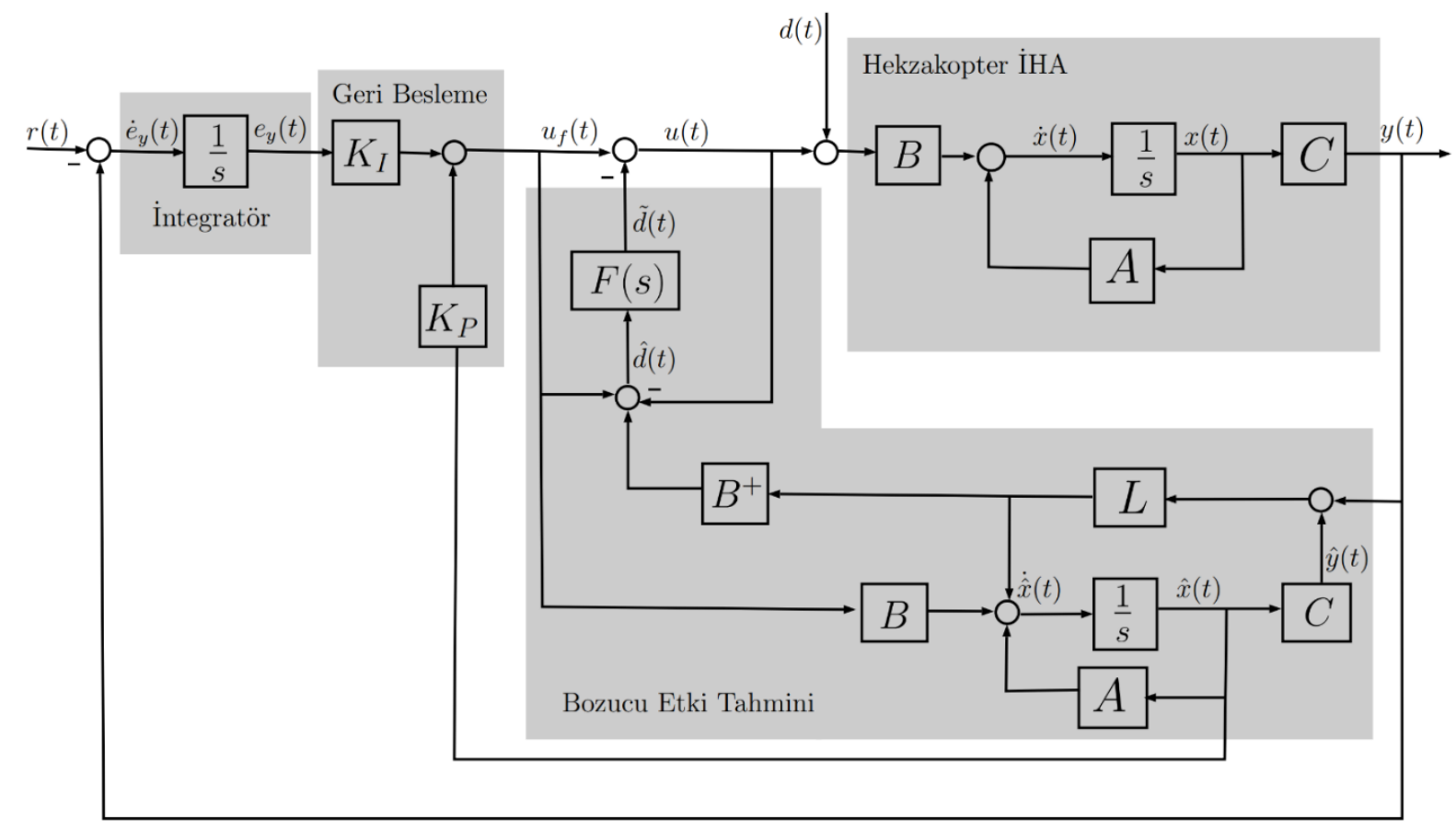

Şekil 2. Girişteki bozucu etkinin tahmini kullanılarak denetleyici tasarım yapısı

$$
\hat{d}(t)=u_{f}(t)-u(t)+B^{+} L(y(t)-C \hat{x}(t))
$$

Burada

$$
B^{+}:=B^{T}\left(B B^{T}\right)^{-1}
$$

ve filtrelenmiş bozucu etkinin tahmini:

$$
\widetilde{D}(s)=F(s) \widehat{D}(s)
$$

Burada $\widetilde{D}(s)$ ve $\widehat{D}(s)$ sembolleri $\tilde{d}(t)$ ve $\hat{d}(t)$ sirasıyla Laplace dönüşümlerini ifade eder. Buradaki $F(s)$ alçak geçiren filtre olup aşağıdaki formdadır:

$$
F(s)=\frac{1}{t_{F} s+1} I_{n}
$$

Parametre $t_{F}$ bozucu etkinin dışlanması için açısal frekans bandına göre belirlenir ve

$$
t_{F} \leq \frac{1}{5 \sim 10} \frac{1}{\omega_{r}}
$$

Burada $\omega_{r}$ bozucu etkiyi dışlamak için en yüksek seçilmiş açısal frekanstır. Denklem (45)'deki tahmin edilen bozucu etki ile denetim kuralı birleştirilirse, aşağıdaki denetim kuralı yazılabilir:

$$
u(t)=u_{f}(t)-\tilde{d}(t)
$$

$\mathrm{Bu}$ denetim kuralı bozucu etkilerin dışlanmasında performansı iyileştirir. Şekil 2'de eşdeğer bozucu etki tahmini kullanılarak bozucu etki önleyici denetleyici tasarımı yapısı verilmiştir.

\subsubsection{Kararlılık Analizi}

Kararlılık analizi iki bölümden oluşur: geri-besleme kazancı $K=\left[\begin{array}{ll}K_{I} & K_{P}\end{array}\right]$, gözlemci kazancı $L$ ve alçak geçirgen filtre $F(s)$ (ayırma teorisi [17]). Elde edilmiş durum geri besleme kazancı $K$ için, gözlemci kazancı $L$ ve alçak geçirgen filtre $F(s)$ kapalı-döngü sistem kararlılığını etkilememelidir. Bunu analiz için sistemde $r(t)=$ $0, d(t)=0$ ve $\Delta x(t)=\hat{x}(t)-x(t)$ olursa doğrusal zamanla değişmez sistemi tekrar yazacak olursak;

$$
\dot{x}(t)=A x(t)+B u(t)
$$

Denklem (48), (42) ve (47) ile birleştirilirse, Denklem (49) elde edilir.

$$
\triangle \dot{x}(t)=(A-L C) \triangle x(t)+B \tilde{d}(t)
$$

Diğer taraftan Denklem (43)'ün eşdeğeri:

$$
\hat{d}(t)=-B^{+} L C \triangle x(t)+\tilde{d}(t) .
$$

Sinyal $\tilde{d}(t)$ 'dan $\hat{d}(t)$ 'e transfer fonksiyonu Denklem (49)(50) kullanılarak Denklem (51) elde edilir.

$$
\begin{aligned}
& G(s)=1-B^{+} L C(s I-(A-L C))^{-1} B \\
& =B^{+}(s I-A)(s I-(A-L C))^{-1} B
\end{aligned}
$$

Uygun durum geri-besleme kazancı $K=\left[\begin{array}{ll}K_{I} & K_{P}\end{array}\right]$ varsa ve eğer

- Sistem matrisleri $(A, B, C)$ kontroledilebilir ve gözlemlenebilir ise,

- $\|G(j \omega) F(j \omega)\|_{\infty}<1, \forall \omega \in[0, \infty)$ ise,

- $A-L C$ kararli ise 
küçük kazanç (small gain) teoremi [18]'den dolayı Denklem (47)'de verilen denetim kuralı sistemin kararlılığını garanti eder.

Önerilen bozucu etki önleyici denetleyicisi tasarımı, aşağıda verilen adımlar uygulanarak yapılır:

- $K=\left[\begin{array}{ll}K_{I} & K_{P}\end{array}\right]$ kazancı LQR yöntemi ile hesaplanır.

- Bozucu etkileri dişlamak için en yüksek açısal frekans $\omega_{r}$ ve buna karşılık gelen $F(s)$ seçilir.

- $\quad A-L C$ kararlı yapmak için gözlemci kazancı $L$ hesaplanır.

- Gözlemcinin kutupları $(A-L C$ 'nin kutupları) durum geri-besleme kazancı ile kapalı döngü sistemin kutuplarından daha solda olmalıdır.

- $\quad 1 / G(j \omega)$ ve $F(j \omega)$ 'nin Bode diyagramı çizilir ve Denklem (52)'deki şartı sağlayıp sağlamadı̆̆ kontrol edilir eğer sağlamıyorsa gözlemci kazancı bu şartı sağlayana kadar tekrar hesaplanır.

$$
\|G(j \omega) F(j \omega)\|_{\infty}<1, \omega \in[0, \infty)
$$

\section{Denetim tasarımı ve benzetim sonuçları}

$\mathrm{Bu}$ bölümde bozucu etki tahmini kullanılarak denetim kuralının insansız hava aracı modeli için etkinliği gösterilecektir. Benzetim çalışmaları Matlab2017b/Simulink ortamında gerçekleştirilmiştir. Önerilen bozucu etki önleyici denetim tasarımın yönelim takip performansı standart denetleyicinin performansı ile karşılatılmıştır.

Burada takip referans sinyalleri adim sinyallerin birleşiminden oluşmakta ve sinyal üretici tarafindan elde edilmektedir. Bozucu etki sistemin yunuslama torkuna etki edecek şekilde sistemin girişine eklenmiştir ve Denklem (53)'deki formda verilmiştir:

$$
d(t)=\left\{\begin{array}{c}
0, \quad t>0 \\
d_{1}(t)+d_{2}(t), \quad t \geq 5
\end{array}\right.
$$

Burada

$$
\begin{aligned}
d_{1}(t) & =9.6 \sin 0.2 \pi(t-5)+4.2 \sin 0.4 \pi(t-5) \\
d_{2}(t) & =\sin 0.6 \pi(t-5) .
\end{aligned}
$$

İlk olarak, bozucu etki ihmal edilerek uygun denetim tasarımı yapılacaktır. İHA modelinin durumlarını ve takip hatalarının integrallerini içeren artırılmış durum modeli Denklem (54) gibi elde edilir.

$$
\left|\begin{array}{c}
\dot{x}(t) \\
\dot{e}(t)_{y}
\end{array}\right|=\left|\begin{array}{cc}
A & 0 \\
-C & 0
\end{array}\right|\left|\begin{array}{c}
x(t) \\
e_{y(t)}(t
\end{array}\right|+\left|\begin{array}{c}
B \\
0
\end{array}\right| u(t)
$$

burada $x=\left[\begin{array}{lllllll}z_{I} \phi & \theta & \psi & \dot{z}_{I} & \dot{\phi} & \dot{\theta} & \dot{\psi}\end{array}\right]^{T}$ durum vektörü, $e_{y}=$ $\left[\begin{array}{llll}e_{z I} & e_{\phi} & e_{\theta} & e_{\psi}\end{array}\right]^{T}$ hata vektörü ve $u=\left[\begin{array}{llll}u_{1} & u_{2} & u_{3} & u_{4}\end{array}\right]^{T}$ giriş vektörüdür. Tablo 1 'de verilmiş olan İHA modelinin fiziksel parametreleri kullanılarak Denklem (33) ile $A$ ve $B$ matrislerinin sayısal değerleri,

$$
A=\left|\begin{array}{ll}
0_{4 \times 4} & 1_{4 \times 4} \\
0_{4 \times 4} & 0_{4 \times 4}
\end{array}\right|
$$

ve

$$
B=\left|\begin{array}{c}
0_{4 \times 4} \\
\operatorname{diag}(0.6515,24.3309,24.3309,16.6945)
\end{array}\right|
$$

şeklinde hesaplanır. Yuvarlanma-yunuslama-yönelme açıları ve aracın denge noktasından yüksekliği $(\phi, \theta, \psi, z)$ sistemin çıkışı olduğu farz edilirse, çıkış matrisi $C=$ $\left|\begin{array}{ccc}0_{3 \times 1} & I_{3 \times 3} & 0_{3 \times 3} \\ I & 0_{1 \times 3} & 0_{1 \times 3}\end{array}\right|$ şeklinde elde edilir.

LQR yöntemi ile geri beslemeli denetimin kazancı hesaplamak için öncelikle ağırlık matrisleri Bölüm 2.2.1.1'deki yöntem kullanılarak Denklem (55)'deki gibi seçilir:

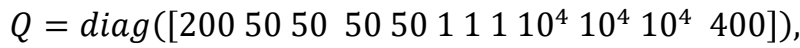

$$
\begin{aligned}
& R=\operatorname{diag}([0.1,0.1,0.1,0.1])
\end{aligned}
$$

Performans indeksi, $J_{K}=\int_{0}^{\infty}\left(x_{a}^{T} Q x_{a}+u^{T} R u\right) d t$ burada $x_{a}=\left[\begin{array}{ll}x & e_{y}\end{array}\right]^{T}$ optimizasyon yapılarak $K$ kazanç matrisi aşağıdaki gibi hesaplanır:

Sonra, bozucu etki sinyalinin en yüksek açısal frekans birleşeni $\omega_{r}=6 \pi \mathrm{rad} / \mathrm{s}$ ve $F(s)$ filtresinin zaman sabiti $t_{F}=0.005 \cong 1 / 10 \omega_{r}$ seçilir. Böylece alçak geçiren filtre Denklem (57) gibi elde edilir.

$$
F(s)=\frac{1}{0.005 s+1}
$$

Ardından performans indeksi $J_{L}=\int_{0}^{\infty}\left(x^{T} Q_{L} x+\right.$ $\left.u^{T} R_{L} u\right) d t \quad$ optimizasyon yapılarak gözlemci kazancı $L$ hesaplanır. Bunun için ağırlık matrisleri $Q_{L}=B^{T} B$ şeklinde seçilerek başlanırsa ve Bölüm 2.2.1.1'deki yöntem

\begin{tabular}{|c|c|c|c|}
\hline Parametre & Tanım & Değer & Birim \\
\hline $\mathbf{m}$ & Kütle & 1.535 & $\mathrm{~kg}$ \\
\hline g & Yerçekimi kuvveti & 9.8 & $\mathrm{~m} / \mathrm{s}^{2}$ \\
\hline$l$ & Kol uzunluğu & 0.275 & $\mathrm{~m}$ \\
\hline$I_{x x}$ & $\mathrm{x}$-eksen atalet momenti & 0.0411 & $\mathrm{~kg} \cdot \mathrm{m}^{2}$ \\
\hline$I_{y y}$ & y-eksen atalet momenti & 0,04178 & $\mathrm{~kg} \cdot \mathrm{m}^{2}$ \\
\hline$I_{z z}$ & z-eksen atalet momenti & 0.0599 & $\mathrm{~kg} \cdot \mathrm{m}^{2}$ \\
\hline$d / b$ & İtme ve sürüklenme oranı & 0.1 & - \\
\hline
\end{tabular}
kullanılırsa, ağırlıklar $Q_{L}=10^{6} \operatorname{diag}(0.01,0.01,0.01,080$, $1200,1200,600)$ ve $R_{L}=\operatorname{diag}(1,1,1,1)$ şeklinde seçilir. Sonraki adımda ise $G(j \omega) F(j \omega)$ transfer fonksiyonun Bode diyagramı Şekil 3'de elde edilir. Bu grafik incelendiğinde Denklem (52)'nin sağladığı açıkça görülmektedir ve bundan dolayı kapalı çevrim sisteminin kararlı olduğu sonucuna varilır.

Tablo 1. Hekzakopter modelinin parametreleri [3] 


$K=\left|\begin{array}{cccccccccccc}73.5 & 0 & 0 & 27 & 0 & 0 & 0 & 0 & 0 & 0 & 0 & -63.2 \\ 0 & 53.8 & 0 & 0 & 0 & 3.8 & 0 & 0 & -316.2 & 0 & 0 & 0 \\ 0 & 0 & 53.8 & 0 & 0 & 0 & 3.8 & 0 & 0 & -316.2 & 0 & 0 \\ 0 & 0 & 0 & 55.5 & 0 & 0 & 0 & 4 & 0 & 0 & -316.2 & 0\end{array}\right|$

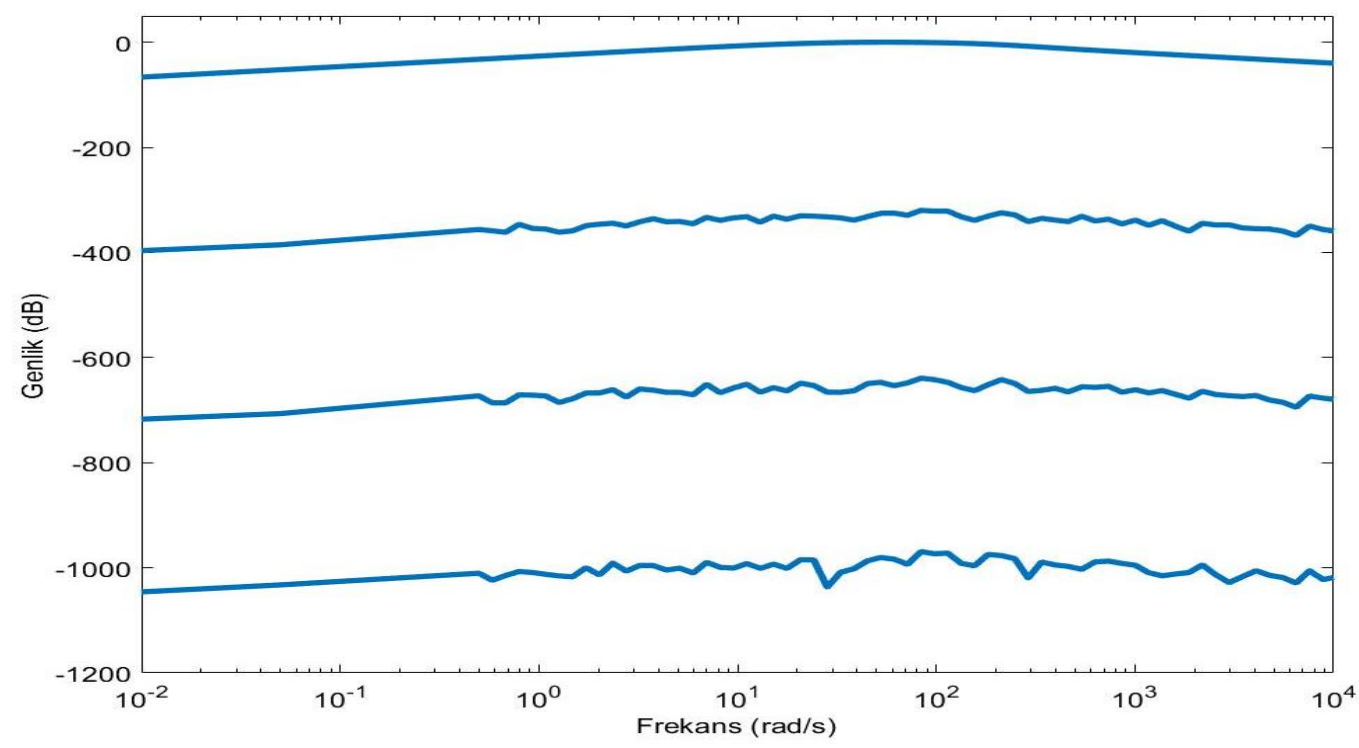

Şekil 3. $\boldsymbol{G}(\boldsymbol{j} \omega) \boldsymbol{F}(\boldsymbol{j} \omega)$ fonksiyonun Bode grafiğgi.

Matlab2017b/Simulink ortamında geliştirilen önerilen denetim yapısı ve İHA modeli Şekil 4' de gösterilmiştir. Şekil 5 'de ise alt sistem bloğunun içyapısı sunulmaktadır. Zaman $t=5$ saniye olduğunda İHA modeli sinüzoidal bozucu etkiye maruz kalmaktadır. Şekil 6'da bozucu etki ve onun tahmini karşılaştırılması verilmiştir. Şekilde de görüldüğü gibi önceden bilinmeyen bozucu etkilerin tahmini oldukça iyi yapılmaktadır.

Standart denetleyicinin yönelim performansı Şekil 7'de gösterilmektedir. Standart denetleyici bozucu etkileri tahmin edecek bir gözlemciye sahip değildir. Bu şekil incelendiğinde, bozucu etkinin yunuslama torkunu etkilemesinden dolayı özelikle yunuslama açısında salınımlar gözlenmektedir. Yunuslama açısındaki sapma $\theta=4^{\circ}$ olarak ölçülmüştür. Daha yüksek bozucu etkiler, örneğin güçlü rüzgâr İHA'nın kararlılığını kayıp etmesine sebep olabilir. Şekil 8'de önerilen bozucu etki önleyici denetleyicinin benzetim sonuçlarını göstermektedir. Burada ise yunuslama açısındaki salınım $\theta=0.2^{\circ}$ olarak ölçülmüştür. $\mathrm{Bu}$ şekilden bozucu etkinin tahmin edilmesi sistem performansını geliştirdiği açıkça görülmektedir.

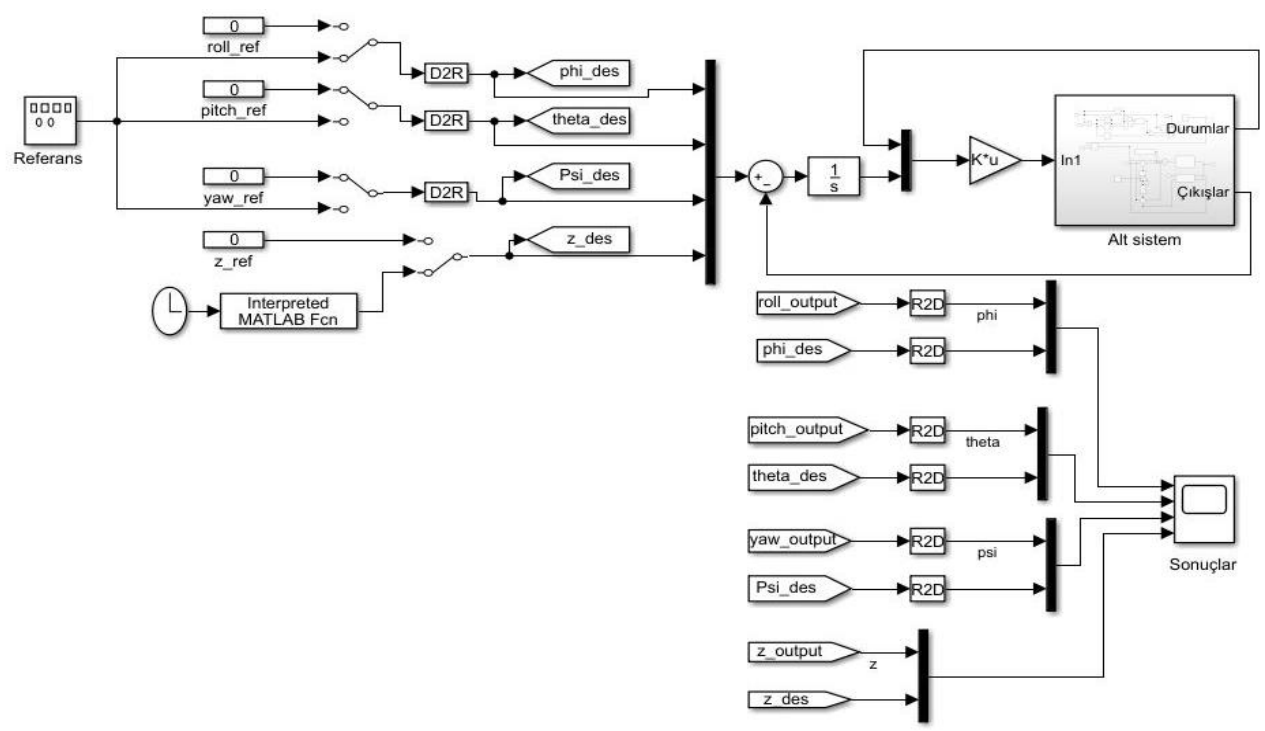

Şekil 4. Matlab2017b/Simulink ortamında geliştirilmiş İHA modeli ve önerilen denetleyici blok diyagramı 

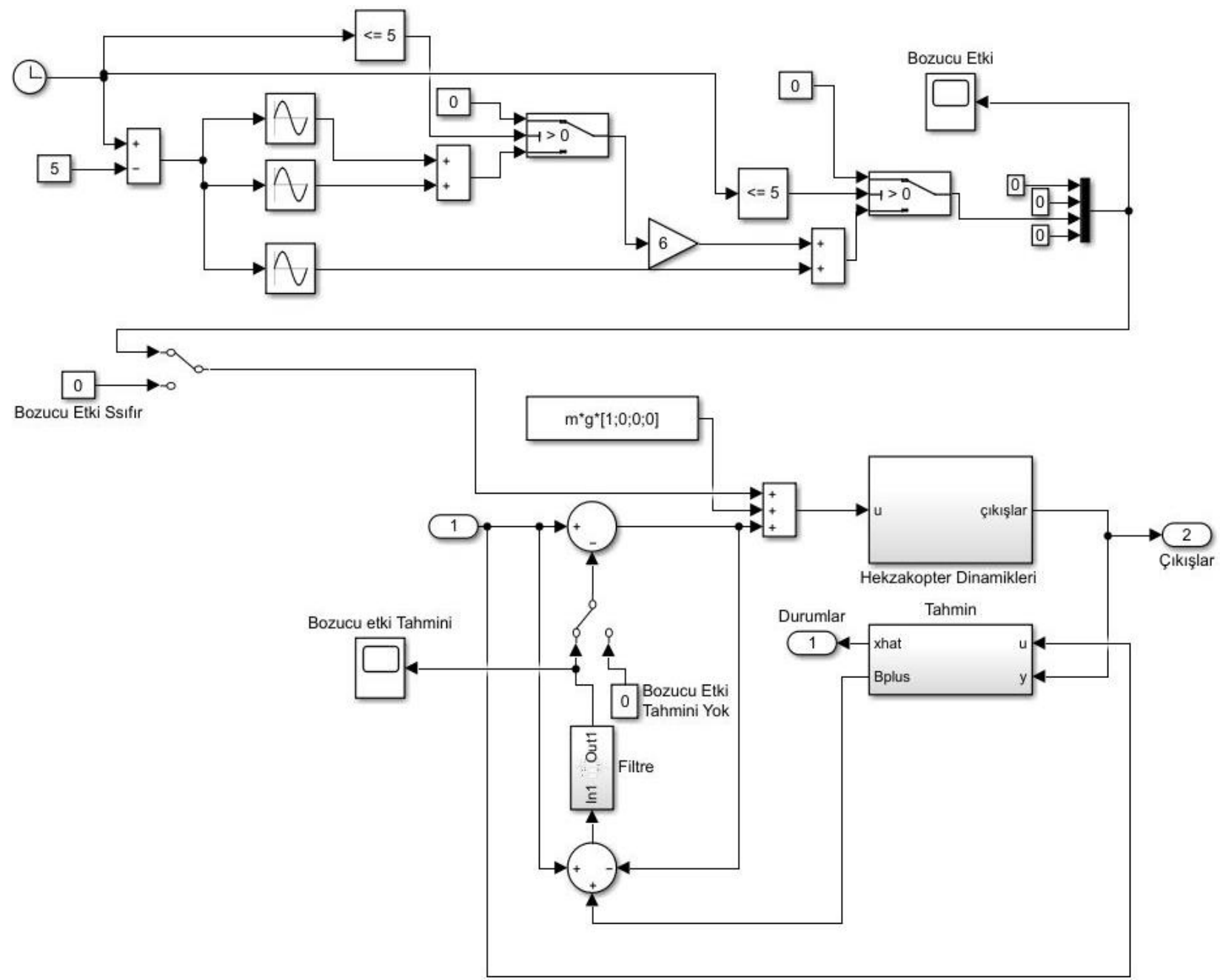

Şekil 5. Alt sistemin içyapı diyagramı

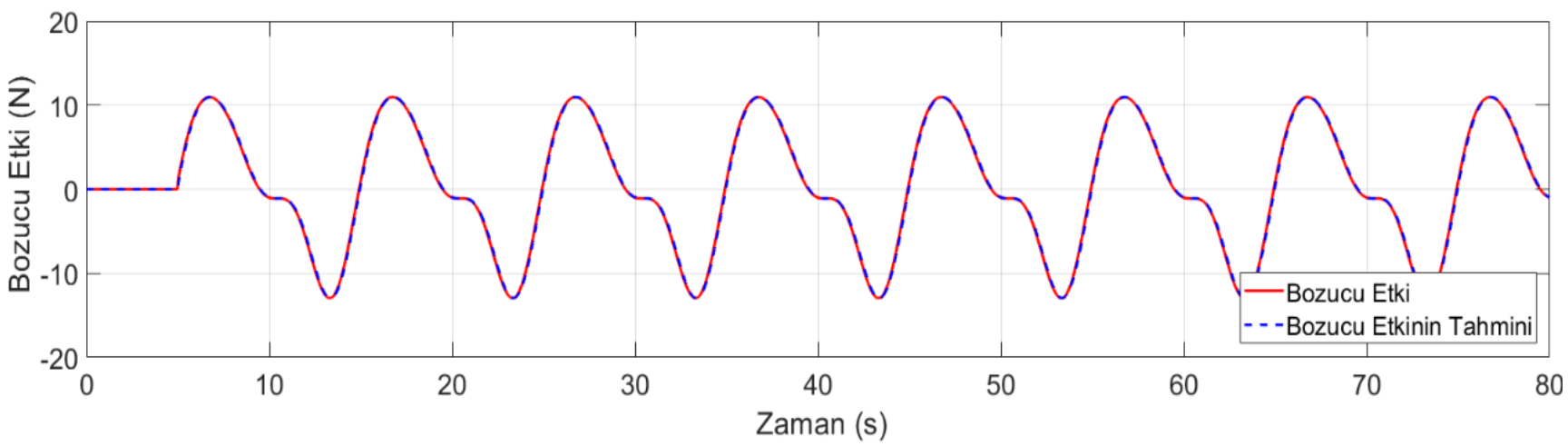

Şekil 6. Bozucu etki $d(t)$ ve bozucu etki tahmininin $\tilde{d}(t)$ karşılaştırması.

Şekil 7 ve 8 karşılaştırıldığında, geliştirilmiş denetleyici tasarımına sahip İHA sisteminin bozucu etkilere maruz kaldığında sistem kararlılığını koruduğu ve referans sinyalini takip performansını iyileştirdiği görülmektedir. Geliştirilmiş denetim kuralı ile yunuslama yönünde yapılan bozucu etkiler elimine edilmiştir. İHA modeli istenilen yüksekliğe çıkmasında her iki denetim yöntemi başarım sağlamıştır. Gözlemci ve alçak geçiren filtreden oluşan mekanizmanın bozucu etkinin tahmin etmesinde gayet başarılı olduğu gözlenmektedir. Sistemdeki belirsizlikler ya da modellenmeyen dinamikler bir bozucu etki olarak düşünülürse aynı yöntem sistemdeki belirsizliğinde üstesinden gelmede de kullanılabilir. Böylece sistemdeki belirsizliklerin etkisi de elimine edilebilir ve sistemin dayanaklığı geliştirilebilir. 

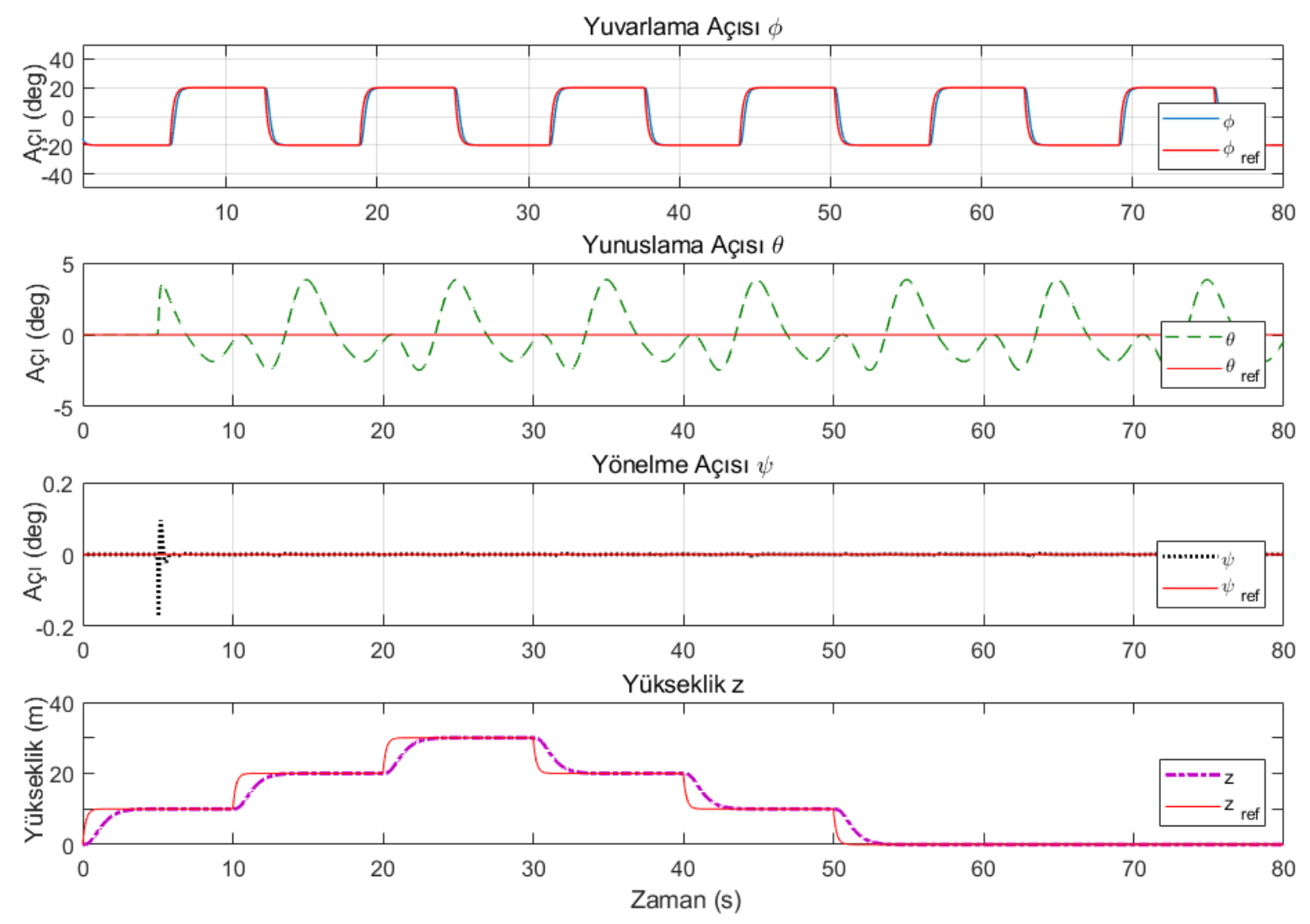

Şekil 7. Bozucu etki tahmini sistemde mevcut değil ve yalnız LQR denetleyici ile benzetim sonuçları.
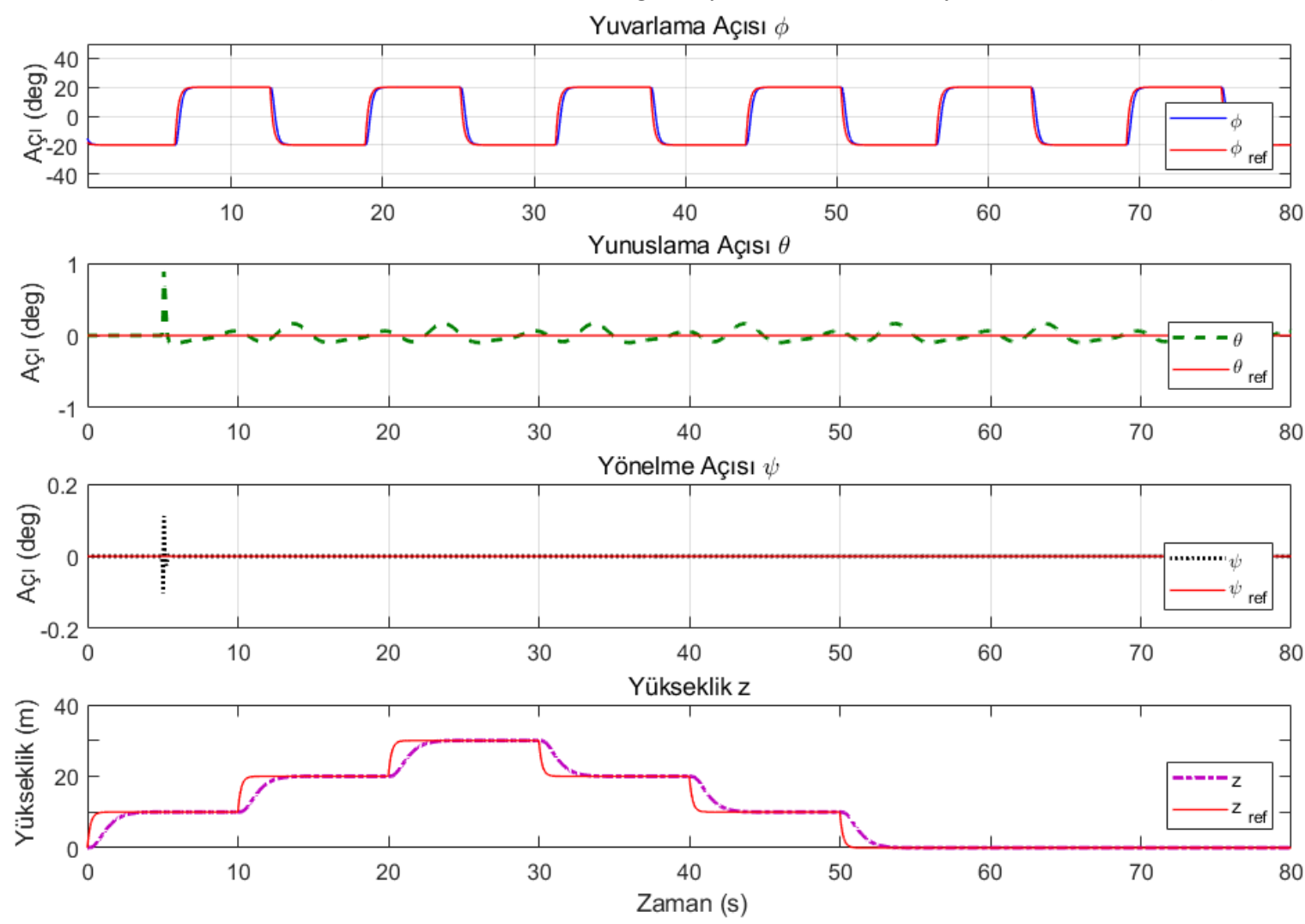

Şekil 8. Bozucu etkilerin tahmini ve LQR denetleyici ile benzetim sonuçları. 


\section{Sonuçlar}

$\mathrm{Bu}$ çalş̧mada durum geri beslemeli bozucu etki önleyici denetleyicinin hekzakopter İHA modeli için benzetim sonuçları verilmiștir. Önerilen denetim tasarımı, standart geri beslemeli denetleyici ile karşılaştırması yapılmıştır. Bozucu etkilerin eşdeğer yaklaşımı kullanılarak İHA modelinin maruz kaldığı bozucu etkiler tahmin edilmiştir. Önerilen bozucu etki önleyici denetim tasarımı standart denetleyiciye göre yunuslama açısında oldukça az salınım elde edilerek sistemin gürbüz kararlılığını korumasını sağlamıştır. Sistemdeki belirsizlikler ya da modellenmeyen dinamikler bir bozucu etki olarak düşünülürse aynı yöntem sistemdeki belirsizliğinde üstesinden gelmede de kullanılabilir. Gelecek çalışma ise farklı bozucu etki önleyici denetleyici tasarlanarak gerçekleştiren tasarımı ile karşılaştırma ve ayrıca bu geliştirilen denetleyicinin gerçek sistem üzerinde uygulanmasına yönelik olacaktır.

\section{Teşekkür}

Bu çalışma, "2019.F13.02.01" nolu proje kapsamında Artvin Çoruh Üniversitesi Bilimsel Araştırma Projeleri Destekleme Programı kapsamında desteklenmektedir.

\section{Çıkar çatışması}

Yazarlar çıkar çatışması olmadığını beyan etmektedir.

\section{Benzerlik oranı (iThenticate): $\% 7$}

\section{Kaynaklar}

[1] A. Kostas, G. Nikolakopoulos, and A. Tzes, Model predictive quadrotor control: attitude, altitude and position experimental studies. IET Control Theory \& Applications, 6.12, 1812-1827, 2012. https://doi.org/ 10.1049/iet-cta.2011.0348

[2] W.H. Chen, J. Yang, L. Guo and S. Li, Disturbanceobserver-based control and related methods-An overview. IEEE Transactions on Industrial Electronics, 63.2, 1083-1095 2016. https://doi.org/10.1109/TIE .20 15.2478397

[3] J. Zhang, D. Gu, C. Deng, and B. Wen, Robust and Adaptive Backstepping Control for Hexacopter UAVs. IEEE Access, 7, 163502-163514, 2019. https://doi .org/10.1109/ ACCESS.2019.2951282

[4] D. Derawi, N. D. Salim, H. Zamzuri, M. A. Abdul Rahman and Nonami, K. Robust attitude control design for a low-cost hexarotor micro aerial vehicle. Transactions of the Institute of Measurement and Control, 38(6), 701-721, 2016. https://doi.org/10. $1177 / 0142331215625768$

[5] A. Alaimo, V. Artale, C. L. R. Milazzo and A. Ricciardello, PID controller applied to hexacopter flight. Journal of Intelligent \& Robotic Systems, 73(14), 261-270, 2014. https://doi.org/10.1007/s10846013-9947-y

[6] A. Freddi, S. Longhi, A. Monteriù and M. Prist, Actuator fault detection and isolation system for an hexacopter. IEEE/ASME 10th International Conference on Mechatronic and Embedded Systems and Applications (MESA) (pp. 1-6), Senigallia, Italy, 2014.

[7] C. Rosales, C. M. Soria and F. G. Rossomando, Identification and adaptive PID Control of a hexacopter UAV based on neural networks. International Journal of Adaptive Control and Signal Processing, 33(1), 749, 2019. https://doi.org/10.1002/acs.2955

[8] J. A. Ligthart, P. Poksawat, L. Wang and H. Nijmeijer, Experimentally validated model predictive controller for a hexacopter. IFAC-PapersOnLine, 50(1), pp. 4076-4081, Toulouse, France, 2017.

[9] N. Tien, D. Saussie, and L. Saydy, Design and Experimental Validation of Robust Self-Scheduled Fault-Tolerant Control Laws for a Multicopter UAV. IEEE/ASME Transactions on Mechatronics, early access, 2020. https://doi.org/10.1109/TMECH .2020 .3042333

[10] K. Guo, J. Jia, X. Yu, L. Guo and L. Xie, Multiple observers based anti-disturbance control for a quadrotor UAV against payload and wind disturbances. Control Engineering Practice, 102, 104560, 2020. https: //doi .org/10.1016/j.conengprac.2020.104560

[11] D. Shi, Z. Wu, and W. Chou, Anti-disturbance trajectory tracking of quadrotor vehicles via generalized extended state observer. Journal of Vibration and Control, 26.13-14, 1173-1186, 2020.https://doi.org/ 10. 1177/1077546319892752

[12] Y. Yuan, L. Cheng, Z. Wang and C. Sun, Position tracking and attitude control for quadrotors via active disturbance rejection control method. Science China Information Sciences, 62(1), 1-10, 2019. https://doi. org/10.1007/s11432-018-9548-5

[13] J. H. She, H. Kobayashi, Y. Ohyama and X. Xin, Disturbance estimation and rejection-An equivalent input disturbance estimator approach. In 43rd IEEE Conference on Decision and Control (CDC), Vol. 2, pp. 1736-1741, Nassau, Bahamas, 2004.

[14] H. Wei, Optimal Robust Control Systems Design and Analysis by State Space Approaches, Ph.D. Thesis, Massey University, Palmerston North, New Zealand, 1995

[15] B. L. Stevens and F. L. Lewis. Aircraft Control and Simulation. John Wiley \& Sons, 1992

[16] X. Chen, W. Cai, M. Wu, and W. Cao, A new approach for periodic disturbance rejection in input-time-delay systems. Transactions of the Institute of Measurement and Control, 40(8), 2589-2598, 2018. https://doi.org/ $10.1177 / 0142331217707571$

[17] B. D. O. Anderson and J. B. Moore, Optimal Control:Linear Quadratic Methods. Englewood Cliffs, NJ: Prentice-Hall, 2007.

[18] K. Zhou, J. C. Doyle, and K. Glover, Robust and Optimal Control. Upper Saddle River, NJ: PrenticeHall, 1996. 